\title{
Still a Small World? University Course Enrollment Networks before and during the COVID-19 Pandemic
}

\author{
Kim A. Weeden, Benjamin Cornwell, Barum Park \\ Cornell University
}

Abstract: In normal times, the network ties that connect students on a college campus are an asset; during a pandemic, they can become a liability. Using prepandemic data from Cornell University, Weeden and Cornwell (2020) showed how co-enrollment in classes creates a "small world" network with high clustering, short path lengths, and multiple independent pathways connecting students. Using data from the fall of 2020, we assess how the structure of the co-enrollment network changed as Cornell, like many other institutions of higher education, adapted to the pandemic by adopting a hybrid instructional model. We find that under hybrid instruction, not only is a much smaller share of students in the face-to-face network, but the paths connecting student pairs in the network lengthened, the share of student pairs connected by three or fewer degrees of separation declined, clustering increased, and a greater share of co-enrollment ties occurred between students in the same field of study. The small world became both less connected and more fragmented.

Keywords: higher education; social networks; enrollment networks; COVID-19

Citation: Weeden, Kim A., Benjamin Cornwell, and Barum Park. 2020. "Still a Small World? University Course Enrollment Networks before and during the COVID-19 Pandemic." Sociological Science 7: XX-YY.

Received: DATERECEIVED

Accepted: DATEACCEPTED

Published: PUBMONTH PUBDAY, PUBYEAR

Editor(s): NAME1, NAME2

DOI: 10.15195/vX.aY

Copyright: @ PUBYEAR The Author(s). This open-access article has been published under a $\mathrm{Cre}$ ative Commons Attribution License, which allows unrestricted use, distribution and reproduction, in any form, as long as the original author and source have been credited. (C) (i)
THE SARS-CoV-2 pandemic is a massive and growing public health crisis, with 1 more than 80 million people infected with COVID-19 and 1.77 million dead worldwide (World Health Organization 2020; data current as of late December). One of the most urgent challenges in stemming the pandemic is to adapt social institutions that are based in face-to-face interactions so they can function safely during a pandemic of a disease that is spread through face-to-face interactions.

A critical first step is to identify aspects of social institutions that give rise to inperson contact networks that increase contagion. To that end, Weeden and Cornwell (2020) analyzed enrollment data from Cornell University in the fall semester of 2019, which was prior to the COVID-19 pandemic, to reveal the structure of the network connecting students to courses, and students to students, through enrollment in classes. This analysis revealed a "small world" network in which nearly all students are connected to each other in the main component of the network, the average path length is short, most randomly chosen pairs of students are connected in three steps or fewer, and students are connected to each other through multiple pathways. Weeden and Cornwell also showed, using simulations, that removing classes of more than 49 students yielded modest but noticeable reductions in the connectivity of the face-to-face enrollment network.

In this article, we assess how the structure of the face-to-face enrollment network changed after Cornell, like many American colleges and universities, adopted a hybrid instructional model — meaning a mix of in-person and online classes-in response to the pandemic. We do so by analyzing new enrollment data from Fall 
2020 ("midpandemic") and comparing them with prepandemic enrollment data from Fall 2019 (Weeden and Cornwell 2020). Our analysis speaks, albeit indirectly, to the epidemiological questions of whether and by how much hybrid instructional models can reduce the risk of viral transmission on a college campus. It also illustrates the fundamental tension facing many institutions of higher education: on one hand, universities' curricular offerings and organizational structure are designed to foster connections between students-a goal that, absent a pandemic, is actively pursued and marketed as one of the key social and educational benefits of learning in the collective setting of a university. On the other hand, the very connections that are an asset in normal times can become a liability during a pandemic, and institutional adaptations to the pandemic perforce alter the structure of these connections.

We find that the connectivity of the enrollment network decreased substantially between the prepandemic and midpandemic semesters. Including nonresidential students, nearly half of Cornell students did not participate in any in-person classes with any other student in Fall 2020. Among students enrolled in face-to-face classes, the enrollment network became more clustered at the local level, paths connecting pairs of students lengthened, fewer pairs of students could reach each other in three steps or fewer, and a smaller share of students was connected to each other by multiple distinct paths. Compared with the prepandemic network, the midpandemic network is also characterized by more homophily with respect to field of study, meaning that a greater share of student-to-student ties in face-to-face classes occurred among students from the same major.

\section{The University Context}

Cornell University is a medium-sized, selective university comprising a mix of endowed and statutory colleges that are part of the State University of New York system. We restrict our analysis to the colleges and instructional programs that are located in Ithaca, New York-Cornell's main campus.

Cornell is among the approximately 21 percent of private and 28 percent of public four-year universities that adopted hybrid instructional models in Fall 2020; another 47 percent of private and 60 percent of public four-year universities planned to be either primarily (but not fully) online or primarily (but not fully) in-person (Chronicle of Higher Education 2020). Although these categories are vaguely defined, the implication is that the majority of American universities ended up with some mix of in-person and online instruction. This shift to hybrid instruction was accompanied, at Cornell and elsewhere, by other adaptations that may have affected enrollment networks, such as requiring physical distance in classrooms, requiring masks (which shifts dynamics in discussion-based classes), and reducing the density of students on campus. ${ }^{1}$

Cornell's instructional model for Fall 2020 was hybrid at the level of class meetings, or "classes" for convenience. A class meeting is one of possibly many components of a single course. For example, Introduction to Sociology is a course consisting of 13 class meetings: a lecture and 12 discussion sections (from which 
each student chooses one). As in Weeden and Cornwell (2020), class meetings constitute one set of nodes in the bipartite network of students and meetings.

In Fall 2020, each class meeting could have its own instructional modality: for example, the lecture could be online, but the discussion section or labs in person. A small percentage (5 percent) were hybrid within the class meeting, meaning that students in the same lecture (for example) rotated between attending in person some days of the week and attending online other days of the week; we treat these as in-person classes. In-person class meetings were effectively capped at 50 students. Some moderately sized classes also reduced the number of students who could enroll, relative to a "normal" year, because physical distancing requirements reduced overall classroom capacity on campus.

Nearly all in-person or hybrid class meetings had online "mirrors," or remote access sections for students who did not to return to Ithaca or were immunocompromised. The exceptions were some in-person class meetings for which there were equivalent online options or that were exempt for pedagogical reasons (e.g., hands-on labs).

Faculty (tenure line and non-tenure track) could choose the modality of their classes based on their personal circumstances, comfort with teaching in person, and pedagogical preferences. Graduate student teaching assistants, many of whom lead discussion sections or labs, could apply for a health exemption. Most but not all departments also allowed teaching assistants to choose their instructional modality freely. Approximately 72 percent of class meetings in Fall 2020 were offered online, and the remaining 28 percent were offered in-person (23 percent) or hybrid (5 percent).

Students could also choose their location. Nearly 18,000 students lived in Ithaca (on campus or off) in Fall 2020, out of approximately 23,800 students who enrolled in at least one class. Students who indicated that they lived in Ithaca ${ }^{2}$ could enroll in classes of any instructional modality. Students who indicated that they lived elsewhere could enroll in-person classes, but they were subsequently shifted into online mirror sections. Of the 18,000 students who lived in Ithaca, approximately 11,700 enrolled in at least one in-person or hybrid class, ${ }^{3}$ meaning they were part of the face-to-face enrollment network.

The data in our analysis mimic the data that Weeden and Cornwell (2020) used to produce the university-wide network for Fall 2019, when almost all Cornell classes were offered in person. ${ }^{4}$ They encompass all class meetings that were taught entirely or partially in person, and all students, including undergraduate, professional, graduate, and nondegree students. ${ }^{5}$ Because we are interested in the face-to-face enrollment network, in our main analysis we restrict the data to students who lived in Ithaca and took at least one in-person class. We also provide some network statistics based on the total number of Ithaca-based students and the total number of students.

Following Weeden and Cornwell (2020), we also differentiate students by their field of study: humanities, arts, and design; social sciences; science, technology, engineering, and math (STEM); interdisciplinary or mixed; undeclared or nondegree programs; and business or law. (See Weeden and Cornwell [2020] for coding details.) 
The assumption is that field of study is a major source of clustering within the enrollment network.

\section{Connectivity within the Enrollment Network}

Table 1 presents measures of the structure of the in-person co-enrollment network at Cornell in the fall terms of 2019 and 2020. Column 1, which is based on Fall 2019 data, replicates the results that Weeden and Cornwell (2020; Table 2) presented for the university-wide network, although we present a slightly different measure of network density and include a measure of field-based assortativity. ${ }^{6}$ Column 2 presents analogous measures for the networks (bipartite and single mode) of Ithaca-based students enrolled in at least one in-person class meeting in Fall 2020. Although we are primarily interested in the comparison of columns 1 and 2, the face-to-face enrollment networks in each of the two years, we also present network measures for Fall 2020 that assume the population at risk for being in the face-to-face enrollment network is all Ithaca-based students (column 3) or all enrolled students (column 4).

Figure 1 depicts the face-to-face enrollment networks in 2019 (Figure 1a; see column 1 of Table 1) and 2020 (Figure 1b; see column 2 of Table 1). ${ }^{7}$ In the diagrams, classes are depicted using light gray squares. Students in different broadly defined fields (arts, humanities, and design; STEM; social sciences; mixed or multidisciplinary; undeclared; and business and law) are depicted using circles of different colors. Enrollments of these students in the classes are represented as gray lines. Broadly speaking, students are positioned near the classes in which they were enrolled and, by extension, near other students who were enrolled in similar classes.

Table 1 and the associated figures show a significant decline in the size of the face-to-face enrollment networks across the two years. In 2019, the in-person coenrollment network included 22,051 students and 6,072 classes; in Fall 2020, it included 11,674 students and 1,735 classes. Similarly, the diagram from Fall 2019 is more densely populated than the diagram from Fall 2020, reflecting the much smaller student population in residence in Fall 2020 and the shift of many classes online.

This shift in the size of the co-enrollment network was accompanied by changes in the structure of the network. In Fall 2020, 94.5 percent of in-person students were connected to each other through classes, compared with 99.1 percent of students in Fall 2019. This is not a large change, but note that the denominator is limited to Ithaca-based students taking at least one in-person class. Among all Ithaca-based students (Table 1, column 3 ), the share is 61.3 percent; among all enrolled students (Table 1 , column 4 ), it is 47.2 percent.

The smaller size of the co-enrollment network also affects the node degrees, or the average number of other students with whom a focal student has direct ties through enrollment in the same class. In Fall 2019, the average Cornell student enrolled in classes with 529 other unique students. Assuming perfect attendance, this means the average student sat in a physical classroom with 529 other students over a week of classes, at a minimum of 50 minutes per class meeting per week 


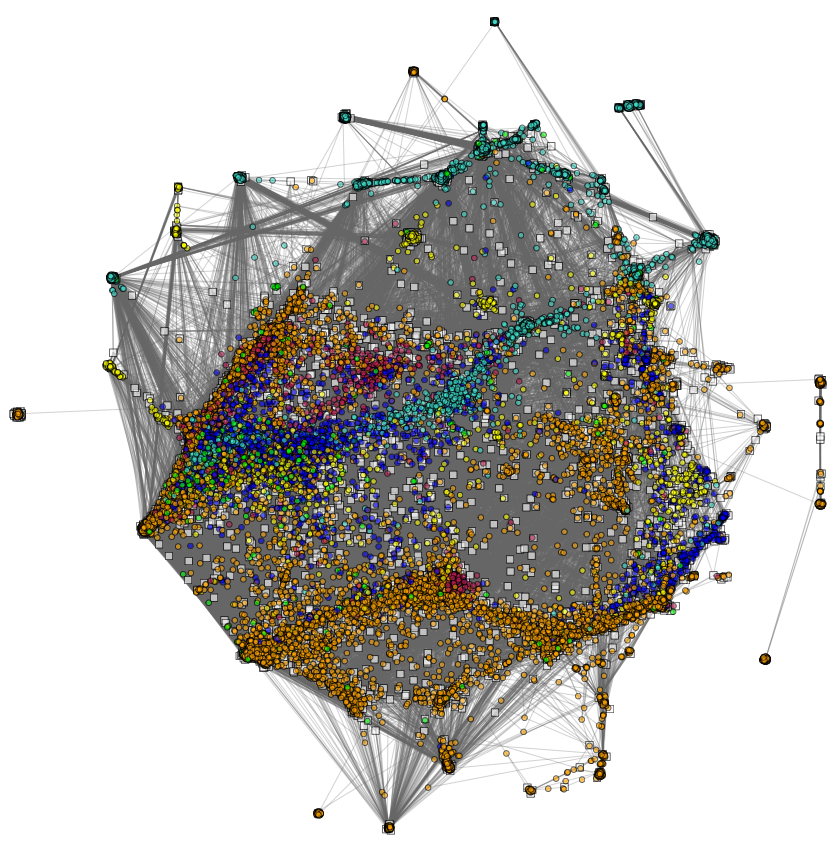

(a) Fall 2019.

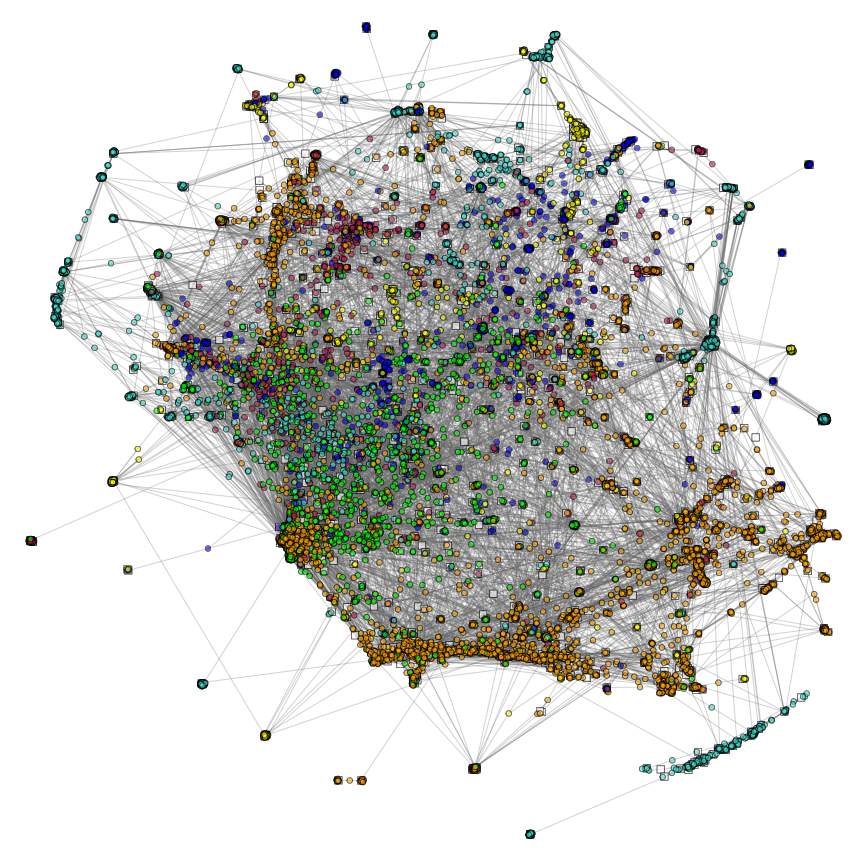

(b) Fall 2020.

Figure 1: Note: The layout for both network plots was generated by the OpenOrd algorithm implement in the igraph package (Csárdi and Nepusz 2006). Students are represented by circles where the colors identify their fields: yellow = humanities, arts, and design; dark blue = social sciences; orange = STEM; rose red $=$ multidisciplinary $/$ mixed; green $=$ undeclared; turquoise $=$ business and law. White squares represent courses. The plots show only the largest component in each of the years. 
Table 1: The Structure of Co-enrollment Networks, 2019 and 2020

\begin{tabular}{lcccc}
\hline & $\begin{array}{c}(1) \\
\text { Fall 2019: } \\
\text { in-person } \\
\text { enrollment } \\
\text { network }\end{array}$ & $\begin{array}{c}\text { Fall 2020: } \\
\text { in-person } \\
\text { enrollment } \\
\text { network }\end{array}$ & $\begin{array}{c}\text { Fall 2020: } \\
\text { students in } \\
\text { residence }\end{array}$ & $\begin{array}{c}\text { Fall 2020: } \\
\text { all enrolled } \\
\text { students }\end{array}$ \\
Measures & & & & \\
\hline Two-Mode (Student-to-Class) Network & 22,051 & 11,674 & 17,986 & 23,376 \\
Number of students (n) & 6,072 & 1,735 & 1,735 & 1,735 \\
Number of in-person classes (m) & 118,314 & 30,637 & 30,637 & 30,637 \\
Number of edges (l) & 0.001 & 0.002 & 0.001 & 0.001 \\
Network density & 0.991 & 0.945 & 0.613 & 0.472 \\
Prop. of students in largest component & 0.976 & 0.911 & 0.911 & 0.911 \\
Prop. of classes in largest component & 0.945 & 0.594 & 0.386 & 0.297 \\
Prop. of students in largest bi-component & 0.730 & 0.843 & 0.843 & 0.843 \\
Prop. of classes in largest bi-component & 0.098 & 0.077 & 0.043 & 0.025 \\
Betweenness centralization & & & & \\
\hline Projected One-Mode (Student-to-Student) Network & $5,832,360$ & 377,603 & 377,603 & 377,603 \\
Network number of unique edges (l) & 0.024 & 0.006 & 0.002 & 0.001 \\
Network density & 0.480 & 0.636 & 0.636 & 0.636 \\
Clustering coefficient (transitivity/closure) & 0.282 & 0.531 & 0.531 & 0.531 \\
Assortativity coefficient (field of study) & 2.466 & 3.345 & 3.345 & 3.345 \\
Average geodesic distance & 10 & 9 & 9 & 9 \\
Network diameter (largest distance) & & & & \\
Proportion reachable in $k$ steps & 0.024 & 0.006 & 0.002 & 0.001 \\
$k=1$ & 0.593 & 0.098 & 0.064 & 0.049 \\
$k=2$ & 0.921 & 0.553 & 0.359 & 0.276 \\
$k=3$ & 0.966 & 0.831 & 0.539 & 0.415 \\
k=4 & & & & \\
\hline N & & & &
\end{tabular}

Note: Data are from Cornell University. With the exception of the assortativity coefficient, which Weeden and Cornwell (2020) did not calculate, all measures in column 1 reproduce their results.

(although most classes involve more than 50 minutes of class time per week). In Fall 2020, the average Cornell student in the face-to-face enrollment network encountered 65 other students in classrooms $(2 \times 377,603 / 11,674 \approx 65$, where 377,603 is the number of edges and 11,674 the number of students).

Additional structural differences in the two terms' enrollment networks derive from the extent to which students are interconnected through multiple, alternative pathways. For example, only 59.4 percent of the in-person students in Fall 2020 are connected to each other through the largest bi-component. The corresponding percentage in Fall 2019 is 94.5 percent. This includes students who have multiple independent pathways connecting them.

Another key difference appears in the average distance between students, which increased by almost an entire step-from 2.47 in 2019 to 3.35 in 2020. Bear in mind that geodesic distance is technically undefined, or infinite, for students who were not taking any in-person classes at all. This increase in distance refers only to 
ties that exist among those who are directly or indirectly connected through the face-to-face enrollment network.

The decline in connectivity in the face-to-face network is more easily conveyed in the estimates of $k$-step reachability (Table 1), which indicate the share of student pairs that are connected through a certain number of steps, or "degrees of separation." In Fall 2019, a given student was likely to enroll in a class with about 2.4 percent of the other students on campus. This number declined to 0.6 percent in Fall 2020. Even more striking is the difference in indirect reachability among the students. In Fall 2019, 59.3 percent of student pairs were indirectly connected through just two steps in the class network; in 2020, this declined to 9.8 percent of student pairs. Similarly, in Fall 2019, 92.1 percent of student pairs were reachable in three steps, and 96.6 percent were reachable in four steps. In Fall 2019, just more than half, 55.3 percent, of student pairs were reachable in three steps, and 83.1 percent of pairs were reachable in four steps.

\section{Field-Based Clustering}

A small-world network is characterized not only by high connectivity but also by high clustering. As we showed in the prior section, the connectivity of the face-toface enrollment network declined substantially between 2019 and 2020. Transitivity of the student-to-student network also increased between 2019 and 2020, indicating that co-enrollment connections between students have become more clustered in general.

This clustering is driven in part by greater within-field assortativity, meaning greater field-based homophily in student co-enrollment ties. To show this, we calculated the Newman's (2003) assortativity coefficient, based on the students' major or graduate degree field, for both 2019 and 2020. This measure compares the observed within-field tie proportion with the expected within-field tie proportion in a counterfactual network where ties are random with respect to field (but the number of ties connected to each field is fixed). ${ }^{8}$ The assortativity coefficient for the one-mode projection in Fall 2019 was 0.282, suggesting a small to moderate tendency toward within-field concentration of ties. In Fall 2020, it nearly doubled to 0.531 , representing a substantial decline in the proportion of ties spanning across the six broad academic fields in our data.

The within-field clustering is visually apparent in Figure 1. In both network graphs, nodes of similar colors tend to lie more closely together than do nodes of different colors. However, the graphs also show some indications that within-field clustering increased in Fall 2020. For example, in the top-right corner of Figure 1a (Fall 2019), students from virtually all fields were densely connected to one another. This heterogeneous cluster of nodes largely disappeared in Figure 1b (Fall 2020). Students still co-enrolled in classes across fields, but cross-field co-enrollment was less common in 2020 than in 2019. 


\section{Conclusion}

Cornell University's shift to a hybrid model of instruction in the Fall 2020 term fundamentally altered the size, connectivity, and clustering of the face-to-face enrollment networks connecting students and classes, relative to the prepandemic enrollment network (Weeden and Cornwell 2020). In Fall 2020, the face-to-face enrollment network connected fewer and a lower share of students because some students chose not to live in Ithaca, chose not to take in-person classes, or lacked the opportunity to take in-person classes given the limited availability of seats in in-person classes. The midpandemic enrollment network, while still a small world, became substantially less connected, with a lower share of students in the main bi-component of the network (where multiple independent paths connect students), longer average path length connecting student pairs, and smaller shares of student pairs connected indirectly in three or four steps.

In addition to a decrease in the general connectivity of student body, the studentto-student network also became more fragmented by field of study. This fragmentation is a predictable, if unintentional, consequence of the shift to hybrid instruction. First, removing the large, in-person classes from the in-person curriculum disproportionately affected introductory courses that are required for several different majors or that fulfill general education distribution requirements, leading to fewer connections among students from different fields. Second, an anticipated shortage of physical classroom space, itself a direct result of public health measures such physical distancing and enhanced ventilation, may have led some departments to reserve in-person spots for their majors.

With these data, we cannot discern how the students' experiences in their faceto-face classes changed in qualitative ways due to smaller in-person classes, less in-person contact with instructors and fellow students, greater within-field homophily, and fewer pairs of students taking multiple in-person classes together. Similarly, we cannot assess how networks outside the classroom changed, whether from other institutional adaptations to the pandemic (e.g., reduced density in the residence halls, take-out dining, restrictions on gatherings, mask-wearing) or as knock-on effects of the hybrid instructional model. It may be, for example, that lower connectivity and greater within-field clustering in the enrollment network fundamentally altered the opportunities for students to form friendships with classmates, in turn affecting social life outside of the classroom. Finally, we cannot show the short- or long-term educational consequences of the changes to the network structure, nor how these changes were experienced unequally across students. These questions, and more, are key tasks for future research. Our results set the stage for this research agenda by showing that a common institutional adaptation to the pandemic turned the "small world" of college enrollment networks into a less connected, more fragmented world.

\section{Notes}


1 To reduce transitions to and from Ithaca, Cornell shifted all courses to online instruction after the Thanksgiving holiday in late November. Our data reflect enrollments during the majority of the time when some courses were in person.

2 As part of Cornell's asymptomatic COVID-19 testing program, students were required to register their physical location even if they lived off campus.

3 Ithaca-based students who did not take in-person classes in Fall 2019 include permanent residents of Ithaca (e.g., many graduate students) and temporary residents who committed to returning before they enrolled in classes (but may have chosen to live locally anyway).

4 Prior to the pandemic, Cornell offered a handful of distance learning courses; these were excluded from Weeden and Cornwell's (2020) analysis.

5 A significant share of the students in the Fall 2019 is also in the Fall 2020 data. However, the structure of the data does not allow us to link students across years.

6 Weeden and Cornwell (2020) expressed density in terms of the relationship between the number of edges and nodes in the overall graph, irrespective of within- and betweenvertex-set possibilities. Here we restrict the denominator to between-vertex-set cells only, which is arguably more intuitive. In practice, this alters the observed density measure by a trivial amount, on the order of 0.001 .

7 Figure 1a is based on the same Fall 2019 data as in Weeden and Cornwell (2020), but a different and more efficient algorithm for mapping the network. It thus looks a bit different from the network diagram in Weeden and Cornwell's Figure 1. Network diagrams in a color scheme that is accessible to readers with some forms of colorblindness are provided in the online supplement.

8 The assortativity coefficient is zero when the within-field tie proportion is equal to the expected proportion under the random mixing scenario. Its upper bound is one, which occurs when all ties are between students of the same field. When the assortativity coefficient is calculated on nominal characteristics of nodes, as we have done here, it can be understood as the modularity of the characteristic-based community structure, scaled to have an upper bound of one.

\section{References}

Csárdi, Gábor, and Tamás Nepusz. 2006. “The igraph Software Package for Complex Network Research." InterJournal 1695. http://www.interjournal .org/manuscript_ abstract.php?361100992.

Chronicle of Higher Education. 2020. "Here's our List of Colleges' Reopening Plans." Chronicle of Higher Education, October 1, 2020. Retrieved November 19, 2020. https: //www . chronicle.com/article/Here-s-a-List-of-Colleges-/248626.

Newman, M. E. J. 2003. “Mixing Patterns in Networks.” Physical Review E 67:026126. https: //doi .org/10.1103/PhysRevE.67.026126.

Weeden, Kim A., and Benjamin Cornwell. 2020. “The Small-World Network of College Classes: Implications for Epidemic Spread on a University Campus." Sociological Science 7(9):222-241. http://doi.org/10.15195/v7 .a9.

World Health Organization. 2020. "Coronavirus Disease (COVID-19) Pandemic." World Health Organization. Retrieved December 29, 2020. https://www . who.int/ emergencies/diseases/novel-coronavirus-2019. 
Acknowledgments: Direct correspondence to Kim A. Weeden, Department of Sociology, Cornell University; kw74@cornell.edu. We acknowledge Cornell University's administration for generously and promptly providing access to anonymized data.

Kim A. Weeden: Department of Sociology, Cornell University. E-mail: kw74@cornell.edu.

Benjamin Cornwell: Department of Sociology, Cornell University. E-mail: btc49@cornell.edu.

Barum Park: Department of Sociology, Cornell University. E-mail: b.park@cornell.edu. 